\title{
Localization of Electromagnetic Radiating Sources in the Near-field of a Uniform Circular Array
}

\author{
Mohd. J Abedin and Ananda Sanagavarapu Mohan \\ Microwave and Wireless Technology Research Laboratory, \\ I \& C Group, Faculty of Engineering, UTS, Australia
}

Tel: +61 29514 7525, Fax: +61 29514 2435, E-mail:\{mabedin,ananda\}@eng.uts.edu.au

\begin{abstract}
In this paper we present a parameter estimation problem for practical electromagnetic signals generated by dipole antennas positioned close to a receiving antenna of uniform circular array (UCA). The positions (direction of arrival and range) and the radiation characteristics of emitting sources are being estimated using super resolution techniques. We also estimate the time difference of arrival (TDOA) to calculate range by using generalized cross correlation of eigenvectors in the signal sub-space. The technique discussed here may be generalized to define a methodology to localize and characterize the radar imaging techniques and may be applicable to the separation of dielectric layers by reflecting signals.
\end{abstract}

Index Terms - Dipole antenna, dielectric layers separation, DOA, radar imaging, TDOA.

\section{INTRODUCTION}

Detection and localization non-invasively, of objects that lie in the near-field of a receiver antenna array is of interest for such applications as microwave imaging and wireless communications. A number of super-resolution algorithms such as MUSIC [1] and ESPRIT [5] have been developed to determine the DOA and distance of sources. Mostly, these techniques are applied to a uniform linear array (ULA). However, there is an ambiguity in DOA estimation using ULA. Another limitation of ULA is the failure to estimate both azimuth and elevation angles which is quite significant for practical localization. Further, most of the existing approaches mainly deal with localization of the signal sources within the context of far-field approximation $[1,3$, 4]. The work that was reported on the estimation of the near-field parameters of radiating sources mostly limited their analysis to the consideration of the point sources.

To attain coverage of $360^{\circ}$ angular plane, a uniform circular array is well suited. All most all the reported work on localization, consider only circular arrays formed of point sources disregarding their polarizations [1]. Here, we consider the case where the incoming electromagnetic signals are radiated from dipole antennas and impinging on a UCA receiver antenna. Use of the MUSIC algorithm to characterize this case requires exhaustive searches over the angular and range plane. This procedure becomes computationally more expensive if we have to estimate both azimuth and elevation angles. Thus, in this paper we employ ESPRIT to reduce the computational burden due to searching.

Accordingly, we employ UCA-ESPRIT $[5,6]$ to estimate the bearing angles of a radiating dipole source which is located in the near-field of an UCA. The UCA-ESPRIT is fundamentally different from standard ESPRIT because UCA does not posses the displacement invariance required by ESPRIT algorithm [5]. Both UCA-ESPRIT and ESPRIT follow the same least square (LS) procedure to estimate bearing angles. Geometrical information of the source location is incorporated in the time delay of the signal received at the receiving antenna. Thus, here, the distance of the source from UCA can be calculated from estimated time difference of arrival (TDOA) of incoming signals.

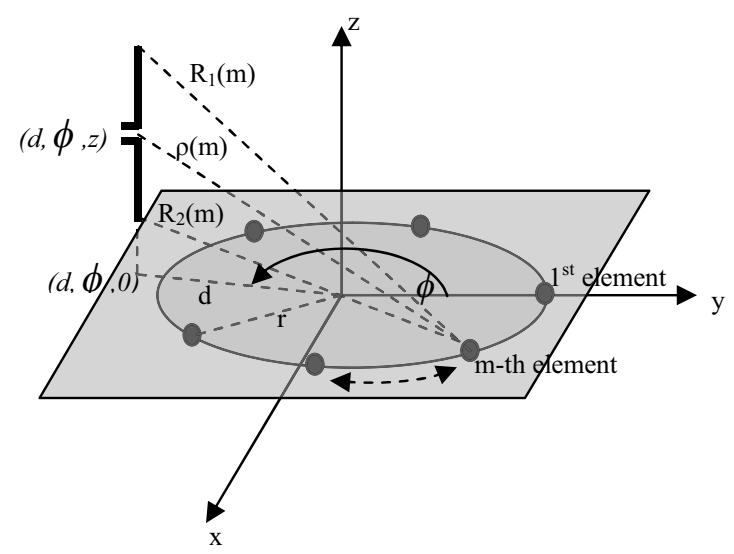

Fig. 1: Near-field scenario with dipole radiating sources. 


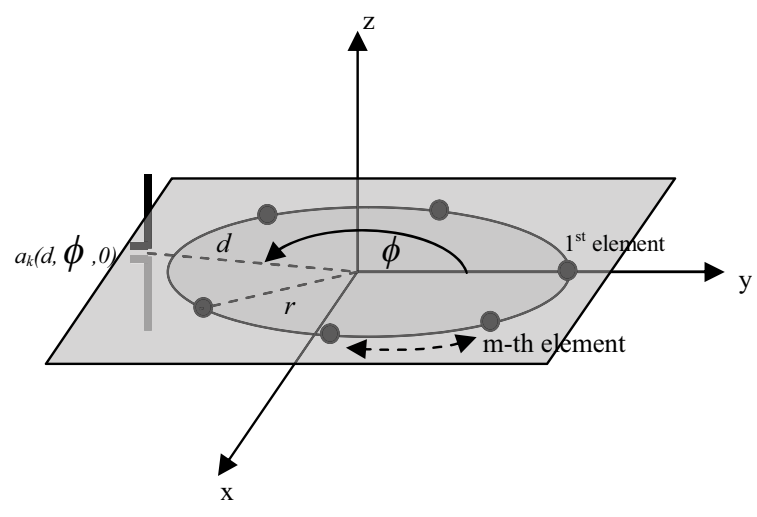

Fig.2: Near-field dipole source geometry lying in the same plane as of UCA.

\section{SIGNAL STUCTURE}

The configuration of UCA is as shown in Fig. 1, where the antenna elements are assumed to be identical and omnidirectional elements which are uniformly distributed over the circumference of a circle of radius $r$. The antenna is considered at $\mathrm{x}-\mathrm{y}$ plane. We assume $k$ radiation sources and consider each one to be a finite thin electric dipole, of length ' $2 \mathrm{~L}$ ' which are located in the near-field of the receiving UCA. The electromagnetic field components can be derived by assuming sinusoidal current distribution on the dipole. Current density J, on a finite thin dipole is directed along the $\mathrm{z}$ direction as shown in Fig. 1, this $J=J_{z}$ only contribute to the magnetic vector potential. Here, we need to evaluate the electric field $E_{z}$, although from the vector potential all the components of the electric field can be obtained.

The signal from $k$-th dipole source at $a_{k}(d, \phi, z)$ is impinges on $m$-th element of UCA. The current component of radiated electric field is $I(t)=I e^{j w t}$. Central feed point of dipole Sources and the UCA are assumed to be in the same plane. The $\mathrm{z}$ component of the electric field can be written as [1]

$$
\begin{aligned}
E_{z}(r(m), t)= & -j \frac{Z_{0}}{4 \pi} I(t)\left[\frac{e^{-j \beta R_{1}(m)}}{R_{1}(m)}+\frac{e^{-j \beta R_{2}(m)}}{R_{2}(m)}\right. \\
& \left.-2 \cos (\beta L) \frac{e^{-j \beta \rho_{k}(m)}}{\rho_{k}(m)}\right] \\
\tau_{k}(m)= & \frac{1}{c} \rho_{k}(m)=\frac{1}{c}\left|d_{k}-r(m)\right|
\end{aligned}
$$

where $c$ is the velocity of propagation of the radiated fields. We can derive

$$
\begin{gathered}
R_{1}(m)=\operatorname{sqrt}\left(\tau_{k}(m)^{2} c^{2}-\left(z_{k}-L\right)^{2}\right) \\
R_{2}(m)=\operatorname{sqrt}\left(\tau_{k}(m)^{2} c^{2}+\left(z_{k}+L\right)^{2}\right)
\end{gathered}
$$

where $r(m)$ is the distance between the centre of UCA to the $m$-th element, $Z_{0}$ is the free space impedance, $120 \pi$, and $\beta$ is the wave number. The signal components and the steering vector at $m$-th element can be found from (1) as

$$
\begin{gathered}
s(t)=-j \frac{Z_{0}}{4 \pi} I(t) \\
a_{m}(d, \phi, z)=\frac{e^{-j \beta R_{1}(m)}}{R_{1}(m)}+\frac{e^{-j \beta R_{2}(m)}}{R_{2}(m)} \\
-2 \cos (\beta L) \frac{e^{-j \beta \rho_{k}(m)}}{\rho_{k}(m)}
\end{gathered}
$$

Similarly, the $\theta$ component of electric field of a vertical electric dipole at UCA in spherical coordinate system can be written as

$$
\begin{gathered}
E_{\theta}(r, t)=-j \frac{Z_{0} L}{4 \pi} I(t) \frac{e^{-j \beta \rho_{k}(m)}}{\rho_{k}(m)}\left[1+\frac{1}{j \beta \rho_{k}(m)}\right. \\
\left.-\frac{1}{\beta^{2} \rho_{k}(m)^{2}}\right]
\end{gathered}
$$

When the dipole is coplanar Fig. 2 with the circular array, the steering vector becomes

$$
\begin{gathered}
s(t)=j \frac{Z_{0} \beta L}{4 \pi} I(t) \\
a_{m}(d, \phi, 0)=-\frac{e^{-j \beta \rho_{k}(m)}}{\rho_{k}(m)}\left(1+\frac{1}{j \beta \rho_{k}(m)}\right. \\
\left.-\frac{1}{\beta^{2} \rho_{k}(m)^{2}}\right)
\end{gathered}
$$

Here, we have considered the radiating sources to be located around the UCA. We assume that they are all excited with the currents of same magnitude with a time delay. Therefore the received signals at UCA also maintain the phase difference information among the incoming signals. The received signals are of the form

$$
\begin{gathered}
\mathbf{x}(t)=\sum_{k=1}^{K} s\left(t-\tau_{k}\right) b_{k} \exp \left(j \varphi_{k}\right) a_{k}(r, \phi, z) \\
+n(t)
\end{gathered}
$$

where $b$ and $\varphi$ are the magnitudes and phase shift of incoming signals. The noise process $n(t)$ is assumed to be an additive zero-mean Gaussian 
distributed. The received data model with narrow band signals can be formulated as

$$
\begin{gathered}
\mathbf{X}=\mathbf{S} \Psi \mathbf{A}^{T}+\mathbf{N} \\
\mathbf{S}=\left[\mathbf{s}\left(t-\tau_{1}\right) \ldots \ldots \ldots \ldots \ldots . \mathbf{s}\left(t-\tau_{1}\right)\right] \\
\boldsymbol{\Psi}=\operatorname{diag}\left[b_{1} e^{j \varphi_{1}} \ldots \ldots \ldots \ldots b_{K} e^{j \varphi_{K}}\right] \\
\mathbf{A}=\left[\mathbf{a}_{1}(r, \phi, z) \ldots \ldots \ldots \ldots \mathbf{a}_{K}(r, \phi, z)\right] \\
\mathbf{N}=\left[\mathbf{n}_{1}(t), \ldots \ldots \ldots \ldots \ldots . \mathbf{n}_{K}(t)\right]^{T}
\end{gathered}
$$

where $\mathbf{A}$ is the $M \times K$ angle of arrival (AOA) matrix having elements space manifold vector a along its column. $\mathbf{S}$ is the incoming signal matrix composed of complex valued signal components $s(t)$. The subscript $T$ denotes transpose of matrix.

\section{DIPOLE SOURCE LOCALISATION}

This section presents a dipole source localization which is assumed to be in the same plane $(z=0)$ of UCA in Fig. 2. To estimate the azimuth angle we use a UCA-ESPRIT algorithm [5], and auto and cross correlation procedure are used to determine the TODA. The distance between the centre of UCA and centre of dipole source can be calculated by solving algebraic equations.

The array output covariance matrix

$$
\begin{aligned}
\mathbf{R}= & E\left[\mathbf{X}(t) \mathbf{X}^{H}(t)\right] \\
& =\mathbf{A} \mathbf{P} \mathbf{A}^{H}+\sigma^{2} \mathbf{I}
\end{aligned}
$$

where $\mathbf{P}=E\left[\mathbf{S}(t) \mathbf{S}^{H}(t)\right]$, a $K \times K$ signal covariance matrix. Performing eigen-value decomposition to the covariance matrix $\mathbf{R}$, the eigen-values can be ordered as $\lambda_{1} \geq \lambda_{2} \ldots \ldots . \geq \lambda_{\mathrm{M}}$ and the corresponding eigenvectors can be denoted by $\boldsymbol{e}_{1}, \ldots \ldots \ldots \boldsymbol{e}_{k}, \boldsymbol{e}_{K+1}, \ldots \ldots \ldots \boldsymbol{e}_{M}$. Here $M-K$ smallest eigenvalues of $\mathbf{R}$ are equal to $\sigma^{2}$. The signal and noise subspace from the eigenvectors can be defined by using MDL criterion using the corresponding eigenvalues. Accordingly, from eigenvalue decomposition, the matrix $\mathbf{U}=\left[\boldsymbol{e}_{1}, \ldots \ldots \ldots \boldsymbol{e}_{k}\right]$ and $\mathbf{V}=\left[\boldsymbol{e}_{K+1}, \ldots \ldots \ldots \boldsymbol{e}_{M}\right]$ spans the estimated signal and noise subspace respectively. Information of time delay among incoming signals and bearing angles of sources are contained in matrix $\mathbf{U}$.

Thus TDOA between the signals can be estimated via the auto and cross correlation of the vectors in $\mathbf{U}$.
Normalized cross correlation of the vectors $\mathbf{u}_{\mathrm{i}}$ and $\mathbf{u}_{\mathrm{j}}$ is given by [3]

$$
R_{i, j}(\tau)=\frac{r_{i, j}(\tau)}{\sqrt{r_{i, i}(0) r_{j, j}(0)}}
$$

where the $r_{i, j}(\tau)$ is the cross correlation of $u_{i}(n)$ and $u_{j}(n)$ and

$$
r_{i, j}\left(\tau_{k}\right)=\frac{1}{N_{s}-\tau_{k}} \sum_{n=1}^{N_{s}-\tau_{k}} u_{i}^{*}\left(n+\tau_{k}\right) u_{j}(n)
$$

where $\tau_{k}=-N_{s}+1, \ldots \ldots \ldots \ldots, N_{s}-1$ and $u_{i}(n)$ is the $\mathrm{n}$-th element of $\mathbf{u}_{\mathrm{i}}$. An estimation of TDOA can be found by maximizing the $R_{i, j}\left(\tau_{k}\right)$. Information of the signal of first arrival can be found from the sign of $\hat{\tau}$.

Again, bearing angles can be obtained from the relation

$$
\hat{\mathbf{\Phi}}=\mathbf{T} \boldsymbol{\Psi} \mathbf{T}^{-1}
$$

Where the eigenvalues of matrix $\Psi$ are equal to the diagonal elements of $\hat{\Phi}$, and the column of $\mathrm{T}$ are the eigenvectors of $\Psi$. Therefore, resulting $\Psi$ are lead to finding $\hat{\Phi} . \Psi$ are determined by applying the least square (LS) procedure but under the noisy environment total least square (TLS) method provides the best results [2]. Now, if $\mu_{k}, k=1,2, \ldots, K$, are the eigenvalues of , the estimates of azimuth angles of radiating sources are given by

$$
\hat{\phi}_{k}=\cos ^{-1}\left(\mu_{k}\right)
$$

Having the estimated azimuth angle and TDOA of emitting source we can calculate the distance of source from the origin of UCA according to (2).

\section{SIMULATION RESULT}

The performance of proposed method was assessed using computer simulations based on UCA with 12 omnidirectional array elements and inter element spacing is equal to half wave length of narrow band electromagnetic signal. Three finite thin dipole sources of length $2 L=\lambda / 2$, are considered at $d_{1}=1.2 \mathrm{~m}, d_{2}=0.8 \mathrm{~m}$ and $d_{3}=1.3 \mathrm{~m}$ whit azimuth angles $\phi_{1}=100^{\circ}, \phi_{2}=250^{\circ}$ and $\phi_{3}=30^{\circ}$ around the UCA respectively. We assumed the radiating sources are at the same plane with receiving antenna array. The number of snapshot is $\mathrm{N}_{\mathrm{s}}=300$, and sample statistics are calculated with 100 independent trials. Simulation results are given in Table I and Fig. 3-5. 
TABLE I

ESTIMATED RESULT FOR THIN DIPOLE SOURCES

\begin{tabular}{|c|c|c|c|c|c|}
\hline \multirow{2}{*}{$\begin{array}{l}\text { Given } \\
\text { Azimuth }\end{array}$} & \multicolumn{2}{|c|}{ Estimated Azimuth } & \multirow{2}{*}{$\begin{array}{l}\text { Given distance } \\
(\mathrm{m})\end{array}$} & \multicolumn{2}{|c|}{ Estimated Distance } \\
\hline & $\begin{array}{c}\text { Mean } \\
\text { Degree }\end{array}$ & $\begin{array}{l}\text { Standard } \\
\text { deviation }\end{array}$ & & Mean $(m)$ & $\begin{array}{l}\text { Standard } \\
\text { deviation }\end{array}$ \\
\hline$\Phi 1\left(100^{\circ}\right)$ & 99.8620 & 1.2060 & $\mathrm{~d}_{1}(1.2)$ & 1.2101 & 0.6842 \\
\hline$\Phi 2\left(250^{\circ}\right)$ & 250.4797 & 0.9144 & $\mathrm{~d}_{2}(0.8)$ & 0.8276 & 0.4520 \\
\hline Ф3 $\left(30^{\circ}\right)$ & 30.3660 & 1.2490 & $\mathrm{~d}_{3}(1.3)$ & 1.2893 & 0.5475 \\
\hline
\end{tabular}

\section{CONCLUSION}

The approach in this paper is much more practical because the signal radiation from dipole sources is considered. This method may be adaptive to find out a complete image of electromagnetic sources. Another important application could be the identification of the position of interference generation and radiation leakage. At the same time UCA is used to exploit the full symmetry of geometry posses by the sources. The UCA-ESPRIT algorithm performs well with this practical problem which is shown with the results computer simulations.

\section{ACKNOWLEDGEMENT}

The work reported in this paper is part of a Discovery Project Grant DP 0346540 funded by the Australian Research Council.

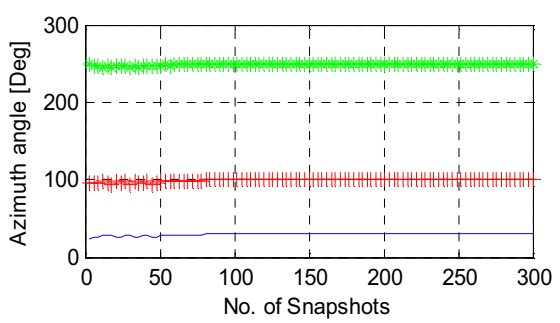

Fig. 3. Estimated azimuth angles $\left(30^{\circ}, 100^{\circ}, 250^{\circ}\right)$ versus number of snapshots.

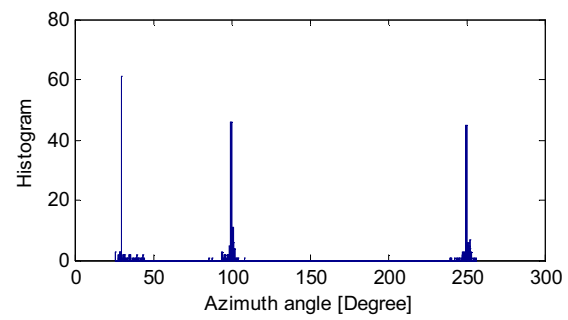

Fig. 4. Histogram of estimated azimuth angles $\left(30^{\circ}, 100^{\circ}, 250^{\circ}\right)$.

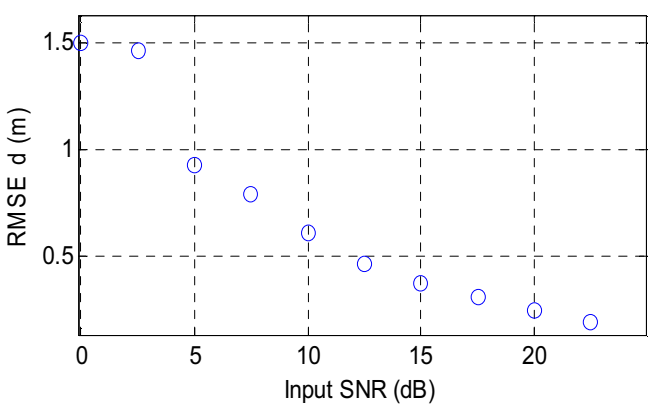

Fig.5. RMS error of estimated distance for different signal to noise ratio.

\section{REFERENCES}

[1] Bruno Audone and Margherita Buzzo Margari, "The use of MUSIC algorithm to characterize emissive sources," IEEE Transaction on Electromagnetic Compatibility, vol. 43, no. 4, pp.688-693, November, 2001

[2] Panayitis Ioannides and Constantine A. Balanis, "Uniform circular array for smart antennas," IEEE Antenna and Propagation magazine, Vol. 47, no. 4, pp.192-206, August, 2005

[3] S. H. Lee, C. S. Ryu and K.K Lee. "Near-field source localization using bottom-mounted linear sensor array in multipath environment" IEE ProceedingsRadar Sonar Navig., vol.149, pp 202-206, 2002.

[4] J.H. Lee, D.H. Park, G.T. Park and K.K. Lee, "Algebraic path-following algorithm for localising 3D near-field sources in uniform circular array," Electronic Letters, vol.39. No. 17, pp. 1283-1285, August, 2003.

[5] Cherian P. Mathews, and Michael D. Zoltowski, "Eigenstructure techniques for 2-D angle estimation with uniform circular arrays" IEEE Transactions on Signal Processing, vol. 42, No. 9, pp 2395-2407, September, 1994.

[6] He Minghao, Yin Yixin and Zhang Xianda, "UCAESPRIT Algorithm for 2-D Angle Estimation," Proceedings of The 5th Int. Conf. on Signal Processing (ICSP2OOO), pp. 437-440, August, 2000. 